\title{
Erratum to: A New Type of Computer Premium and Its Correlation to Individual Wages
}

\author{
Yasuharu UKAI ${ }^{1)}$ \\ 1) The Research Institute for Socionetwork Strategies, Kansai University \\ 3-3-35 Yamate-cho, Suita 564-8680, Osaka, Japan \\ ukai@kansai-u.ac.jp \\ C) Springer Japan 2013
}

Erratum to: Rev Socionetwork Strat (2012) 6:49-63 DOI10.1007/s12626-012-027-8

Unfortunately, the author's affiliation has been incorrectly published. Corrected affiliation of the author is shown above. 